\title{
Optimal Economic Growth under Stochastic Environmental Impact: Sensitivity Analysis
}

Elena Rovenskaya (rovenska@iiasa.ac.at)

\section{Approved by}

Arkady Kryazhimskiy (kryazhim@iiasa.ac.at),

Program Leader, Dynamic Systems Program

Fabian Wagner (wagnerf@iiasa.ac.at),

Co-Leader, Greenhouse Gases Initiative,

January 2008 


\begin{abstract}
In this work we present an approach toward the sensitivity analysis of optimal economic growth to a negative environmental impact driven by random natural hazards that damage the production output. We use a simplified model of the GDP whose growth leads to the increase of GHG in the atmosphere provided investment in cleaning is insufficient. The hypothesis of the Poisson probability distribution of the natural hazards is used at the first stage of the research. We apply the standard utility function - the discounted integral consumption and construct an optimal investment policy in production and cleaning together with optimal GDP trajectories. We calibrate the model in the global scale and analyze the sensitivity of obtained optimal growth scenarios with respect to uncertain parameters of the Poisson distribution.
\end{abstract}

Key words: climate change, natural hazards, GDP growth 


\section{About the Author}

Dr. Elena Rovenskaya (PhD in mathematics) is a junior research scholar at the Faculty of Computational Mathematics and Cybernetics, Moscow State University, Russia and a researcher at the Dynamic Systems Program, International Institute for Applied Systems Analysis, Austria.

\section{Acknowledgments}

The author would like to express her gratitude to Dr. Brian O'Neill, Dr. Fabian Wagner, Dr. Michael Obersteiner and Dr. Oscar Franklin for the interesting ideas, and to Dr. Arkady Kryazhimskiy for fruitful discussions of the mathematical aspects of this study. 


\section{Contents}

1 Introduction $\quad 1$

2 Model 2

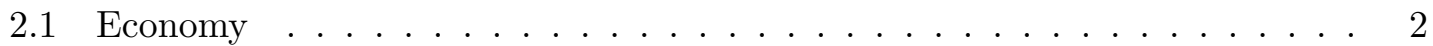

2.2 Natural hazards . . . . . . . . . . . . . . . . . . . . . . . . 3

2.3 Utility . . . . . . . . . . . . . . . . . . . . . . 4

3 Optimal production and optimal cleaning 5

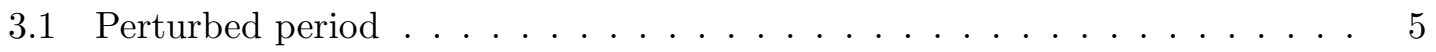

3.2 Pre-perturbed period . . . . . . . . . . . . . . . . 6

4 Global Calibration $\quad 9$

5 Optimal GDP and optimal GHG $\quad 11$

6 Sensitivity Analysis 13

$\begin{array}{lll}7 & \text { Discussion } & 15\end{array}$

$\begin{array}{ll}\text { References } & 18\end{array}$

$\begin{array}{ll}\text { A Proof of Theorem } 1 & 20\end{array}$

B Proof of Lemma $1 \quad 22$

C Proofs of Lemma 3 and Lemma $4 \quad 22$ 


\title{
Optimal Economic Growth under Stochastic Environmental Impact: Sensitivity Analysis
}

\author{
Elena Rovenskaya (rovenska@iiasa.ac.at)
}

\section{Introduction}

Uncertainty arising in assessment of economic growth in relation to climate change create enormous hurdles for scientists, stakeholders and policy makers (see, e.g.,

[Obersteiner et al, 2001]). One of the key issues is how policy choices can balance uncertainty in costs and benefits in situations when one is unsure what constraints on the atmospheric concentration of GHG are sufficient for preventing dangerous interference with the climate system, and what is the degree of danger from exceeding a "safe" level of the GHG concentration.

In this context, a dilemma arises: either to invest in abatement efforts today in order to prevent still unknown negative effects that may or may not occur in the future, or to delay investment until a better knowledge on the feedback between the economy and environment is gained. A basic social goal is to minimize both the social cost of carbon emission and the abatement cost.

Modelers of socio-economic and environmental processes are challenged to create tools for finding optimal strategies for global development under uncertain impact of climate change on human's production. Well-known DICE-type models ([Nordhaus, 1994] and [Nordhaus, Boyer, 2001]) tie up the neoclassical economic growth theory and global warming theory. These models view investment in economy sectors as variable control inputs. Using different investment scenarios, one generates future projections for key economic and environmental indicators. Assuming that a model's parameter values are given, one finds the optimal investment strategy that maximizes the utility social welfare. A number of studies initiated by Nordhaus's approach are aimed at economic assessment of GHG limitation under different types of uncertainty (see [Kainuma, 2006], [Keller et al, 2004], and [Toll, 1994], and also the author's works [Rovenskaya, 2005, Rovenskaya, 2006]).

At this stage of research, it is reasonable to complement the original purely deterministic DICE model by stochastic DICE-type models which could better represent the nature of the environmental impact on the economy. In this context, recent IIASA works, e.g., [O'Neill et al, 2005], should be mentioned. The former develops a simplified stochastic "act then learn" model; and the latter suggests a dynamical multi-stage model assuming that climate provides a stochastic damaging impact on the world capital stock.

The stochastic properties of a feedback of the environmental quality and the economy are being widely discussed nowadays. Such studies as [Keller et al, 2004],

[Hare, Meinshausen, 2006], [Meinshausen et al, 2006] staying in this line are mainly focused on possible distributions of climate sensitivity. [Keller et al, 2004] indicates that "the probability distribution of the threshold-specific damages seems at this time unknown." In this context the analysis of the sensitivity of the model's output to variations in the parameters of the probability distribution may help us understand the degree of the importance of that quantitative information for decision-making. 


\section{Model}

\subsection{Economy}

We consider a one-sector growth model the so called production technology as the key driver of the world economy. Let $T$ stand for the production technology stock used for producing public goods, and $C$ stand for the cleaning technology stock used for barring greenhouse gases emissions that result from human production activity and go to the atmosphere. Let $Y$ be the current GDP value. We assume that the constant fraction of the GDP $u_{*} \in[0,1]$ is yearly available for developing both production and cleaning technologies. The rest fraction of the GDP is consumed by the society. Let $u \in\left[0, u^{*}\right]$ be a time-varying fraction of the GDP yearly allocated for developing the production technology stock whose dynamics is given by

$$
\dot{T}=u Y-\delta T, \quad T(0)=T_{0} .
$$

The dynamics of the cleaning technology stock is given by

$$
\dot{C}=\left(u_{*}-u\right) Y-\delta C, \quad C(0)=C_{0} .
$$

In (1) and (2) $T_{0}>0, C_{0}>0$ are given initial values for $T$ and $C, \delta$ refers to depreciation. In the dynamics $(1),(2)$ the production ratio $u(\cdot)$ is viewed as a time-varying control.

Let $E$ be the greenhouse gases stock accumulated in the atmosphere. We assume that GHG emissions are generated by production and restrained by implementation of cleaning technology. In other words the growth rate of increase of $E$ is positively related to the current production technology stock $T$ and negatively related to the current cleaning technology stock $C$ :

$$
\dot{E}=\max \{\beta T-\gamma C, 0\}, \quad E(0)=E_{0},
$$

where $E_{0}>0$ is a given initial value for $E$. For the reason of simplicity we don't take into account the depreciation. The suggested form for dynamics of the atmospheric GHG implies that the role of the cleaning technology is to decrease a rate of concentration growth but not to decrease the concentration itself: even if we invest all admissible resources in cleaning, the GHG concentration should not decrease.

In further analysis we will distinguish two states of the environment: we will say that the system is functioning in a "safe" mode if the current value of atmospheric GHG $E(t)$ does not exceed a critical level $E_{*}>E_{0}$ and that the system is functioning in an "unsafe" mode otherwise. We will specify the meaning of these terms in the next section.

In order to avoid difficulties with an eventual predominance of cleaning technology stock we assume a gap between $E_{0}$ and $E_{*}$ to be not too big, namely,

$$
E_{*}-E_{0} \leq \frac{\left(\beta T_{0}-\gamma C_{0}\right)^{2}}{2 \gamma u_{*} Y_{0}} .
$$

Remark 1 Given this assumption we have increasing emissions trajectories in a "safe" zone for all admissible controls.

Indeed, let us consider the best-case scenario for emissions, i.e., a control

$$
u(t)=0 \quad(t \geq 0) .
$$

Then

$$
T(t)=T_{0} \quad \text { and } \quad C(t)=C_{0}+u_{*} Y_{0} t
$$


for $t \geq 0$. Emissions become

$$
E(t)=E_{0}+\left(\beta T_{0}-\gamma C_{0}\right) t-\frac{\gamma u_{*} Y_{0} t^{2}}{2} .
$$

In this case $E(\cdot)$ is a quadratic function which increases from $t=0$, approaches its maximum value

$$
E_{\text {max }}=E_{0}+\frac{\left(\beta T_{0}-\gamma C_{0}\right)^{2}}{2 \gamma u_{*} Y_{0}}
$$

at

$$
t=\frac{\beta T_{0}-\gamma C_{0}}{\gamma u_{*} Y_{0}}
$$

and then decreases. Thus letting $E_{*}$ be less then $E_{\max }$ we get increasing trajectories of emissions $E(t)$ for all admissible controls.

Hence if $E(t) \leq E_{*}$ can let

$$
\dot{E}=\beta T-\gamma C, \quad E(0)=E_{0} .
$$

Figure 1 illustrates the set of values of $E_{*}$ and $\gamma$ satisfying to (3).

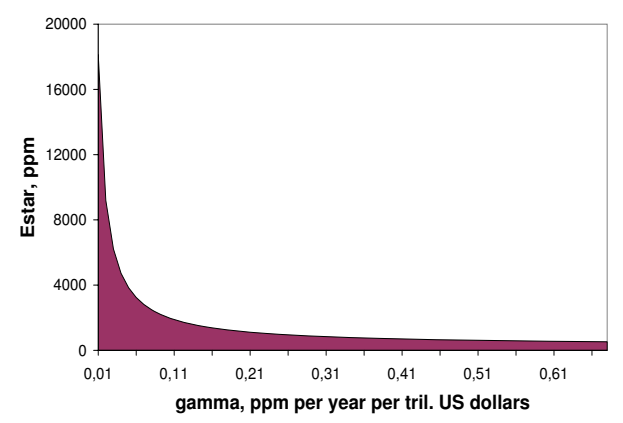

Figure 1: Couples $\left(\gamma, E_{*}\right)$ satisfying to (3) lie in a lilac area.

\subsection{Natural hazards}

In line with numerous speculations and works on modelling the feedback between the environment and economic growth (e.g., [Nordhaus, Boyer, 2001]), we assume the negative impact of the increasing atmospheric GHG on the economy.

Namely we believe that provided the atmospheric GHG stock does not exceed a certain critical level $E_{*}$ the global economy is functioning in a "safe" mode. In this case we assume the simplest form of the Cobb-Douglas production function

$$
Y=A T
$$

where $A$ is an efficiency coefficient which is supposed to be constant on the considered time horizon. In the "safe" mode the aggregated GHG emission is growing due to growing production in accordance with (4). 
However as soon as the GHG stock exceeds the critical limit $E_{*}$ the economy enters an "unsafe" zone in which the climate change issues become significant. We assume the feedback of atmospheric GHG on the economy through global warming and caused by it natural hazards. We guess that in this case yearly the fraction of the GDP equal to $\Omega \in[0,1]$ is damaged by natural hazards caused by climate change which leads to

$$
Y=\Omega A T \text {. }
$$

More specifically we introduce a variable hazard index $\zeta(t)$ that takes value 1 if a hazard occurs at time $t$ and value 0 otherwise. We set

$$
\Omega(t)= \begin{cases}1, & \text { if } \quad E(t) \leq E_{*}, \\ 1, & \text { if } E(t)>E_{*} \text { and } \zeta(t)=0 \\ 0, & \text { if } E(t)>E_{*} \text { and } \zeta(t)=1\end{cases}
$$

We assume that all hazards are equal in strength and set $a \in(0,1]$ be a parameter characterizing a strength of a hazard. Also we assume that at each point in time no more than one hazard may occur. We believe that the hazard index $\zeta(t)$ is a generator of a standard Poisson process describing the evolution of the number of hazards occuring over the expanding time interval $[0, t]$. Namely, for each $t \geq 0$ and each $h \in[0, \tau]$ we denote by $\eta(t, h)$ the number of hazards occuring on the time interval $(t-h, t]$ (or, equivalently, the number if instants $\tau \in(t-h, t]$ such that $\zeta(\tau)=1)$ and assume that for each $j=0,1, \ldots$ the probability for $\eta(t, h)=j$ is given by

$$
P[\eta(t, h)=j]=\frac{(\lambda h)^{j}}{j !} e^{-\lambda h} .
$$

\section{$2.3 \quad$ Utility}

We suppose that the society is guided by the standard utility counting the discounted integral consumption over the infinite ${ }^{1}$ time horizon

$$
\int_{0}^{\infty} e^{-\rho t} \ln \left[\left(1-u_{*}\right) Y(t)\right] d t
$$

or, getting rid from the additive constant, equivalently

$$
J[u]=\int_{0}^{\infty} e^{-\rho t} \ln Y(t) d t .
$$

Due to stochastic nature of $Y(t)$ (see (6) we understand an optimal control problem as follows: find find a piece-wise continuous control $u=u(\cdot): u(t) \in\left[0, u_{*}\right](t \in[0, \infty))$ that maximizes the expected utility, i.e.,

$$
W[u]=\mathbf{E}[J[u]] \rightarrow \max _{u}
$$

under (9) and the dynamics $(1)-(6)$.

Let us specify the form of the expected utility $W$. Obviously the life of system (1) - (6) is split into two periods: the pre-perturbed period $[0, \tau]$ on which $E(t) \leq E_{*}$, catastrophes

\footnotetext{
${ }^{1}$ One can consider this utility on the finite time horizon $[0, \theta]$. All conclusions made in what follows for the infinite time horizon case will remain for the finite time horizon case; formulas will have modified forms explicitly reflecting the quantitative dependence on $\theta$. For the reason of simplicity of representation of the results in this paper we restrict ourselves to the infinite time horizon case.
} 
do not occur and dynamics $(1)-(6)$ is deterministic; and the perturbed period $(\tau, \infty)$ on which $E(t)>E_{*}$ and thanks to random natural catastrophes dynamics (1) - (6) becomes stochastic. Accordingly, we represent controls $u(\cdot)$ in problem (10) as a piece-function of the form

$$
u(t)= \begin{cases}u^{0}(t), \quad t \in[0, \tau] \\ u^{1}(t), \quad t \in(\tau, \infty) .\end{cases}
$$

Consequently the expected utility $W$ can be represented as two additive terms corresponding to these two periods:

$$
W[u]=W\left[\tau, u^{0}, u^{1}\right]=J^{0}\left[u^{0}\right]+\mathbf{E}\left[J^{1}\left[u^{1}\right]\right] .
$$

In other words the optimal economic growth problem requires finding the optimal control on the pre-perturbed period, $u^{0}(\cdot)$, the switching time $\tau$, and the optimal control on the perturbed period, $u^{1}(\cdot)$.

\section{Optimal production and optimal cleaning}

In this section and in what follows we simplify the dynamics of the production and cleaning technology stocks by ignoring technology depreciation, i.e., in (1), (2) $\delta=0$.

\subsection{Perturbed period}

Let us analyze the behavior of system (1) - (6) after $E(t)$ has exceeded the critical level $E_{*}$. It turns out that regardless what is happening on the pre-perturbed period and a time moment when system's dynamics switches from deterministic to stochastic, one finds the optimal control on the perturbed period. Theorem 1 comprises this result.

Theorem 1 Let $u(\cdot)$ be a control optimal in problem (10) of form (11). Let $\bar{u} \leq u_{*}$ be the maximum control admissible for the perturbed period ${ }^{2}$. Then on the perturbed period the optimal control takes its maximum admissible value, i.e.,

$$
u^{1}(t)=\bar{u} \quad(t \in(\tau, \infty)) .
$$

A formal proof of Theorem 1 is given in Appendix A.

The fact that the optimal control in the perturbed period does not depend on the current value of aggregated emissions, $E(t)$, is a consequence of features of Poisson process and the assumption that all hazards are equal in strength. Moreover we see that as soon as the world economy abandons a "safe" zone where no natural hazards driven by industrial GHG occur, there is no economic profit any more (in our model) to prevent a further increase in atmospheric GHG. In other words we assume the environmental impact is insensitive to the level of the aggregated GHG emissions in the "unsafe" area. This rather extreme assumption nevertheless can be accepted on rather middle time perspective ${ }^{3}$.

Because of Theorem 1 we are now aimed at finding an optimal control on the preperturbed period and the switching time $\tau$. Let us remind that $\tau$ is a time moment when aggregated GHG emissions $E(t)$ hits the level $E_{*}$. Namely we have

\footnotetext{
${ }^{2}$ One can choose $\bar{u}$ imposing risks constraints in "unsafe" zone. It is often that a dispersion of a random variable acts as a measure of risks in a stochastic dynamics. Limiting the dispersion of the utility $J$ after the system enters "unsafe" zone one can get additional constraints on the optimal investment policy.

${ }^{3}$ But see suggestions on overcoming this effect in Discussion section.
} 
Problem A: supposing that $u^{1}(\cdot)$ has form (13) and the economy's dynamics is given by $(1)-(6)$ with $\delta=0$, find a couple $\left(\tau, u^{0}(\cdot)\right)$ that

$$
W_{1}\left[\tau, u^{0}\right]=W\left[\tau, u^{0}, u^{1}\right] \rightarrow \max _{\tau, u^{0}(\cdot)}
$$

where $W$ is defined by (12).

The following lemma gives an alternative formula for the utility $W_{1}$ and will allow to simplify problem A.

Lemma 1 Problem A is equivalent to the next optimal control problem

$$
\begin{aligned}
W_{1}\left[\tau, u^{0}\right] & \rightarrow \max _{\tau \geq 0, u^{0}}, \\
\dot{T}(t) & =A u(t) T(t), \quad T(0)=T_{0}, \\
\dot{C}(t) & =A\left(u_{*}-u^{0}(t)\right) T(t), \quad C(0)=C_{0}, \\
\dot{E}(t) & =\beta T(t)-\gamma C(t), \quad E(0)=E_{0}, \quad E(\tau)=E_{*}, \\
u^{0}(t) & \in\left[0, u_{*}\right], \\
(t & \in[0, \tau]),
\end{aligned}
$$

where

$$
W_{1}\left[\tau, u^{0}\right]=\int_{0}^{\tau} e^{-\rho t} \ln A T(t) d t+\frac{e^{-\rho \tau}}{\rho}\left[\ln (A T(\tau))+\frac{A \bar{u}+\lambda \ln a}{\rho}\right] .
$$

We provide a proof to this Lemma in Appendix B.

\subsection{Pre-perturbed period}

In this section we solve problem A, build an optimal control on the pre-perturbed period $u^{0}(\cdot)$ and define the optimal switching time $\tau$ which completes the process of solving problem (10).

First let us specify the deterministic dynamics of the system (1) - (6). In this section for technical reason we eliminate the upper index of a control on a pre-perturbed interval, i.e., instead of $u^{0}$ we will simply write $u$. Since for $t \in[0, \tau]$ we have $\Omega(t)=1$, by $(1)-(6)$ for an arbitrary control $u(t)$ we get

$$
\begin{aligned}
T(t) & =T_{0} e^{A p(t)} \\
C(t) & =C_{0}+u_{*} A T_{0} \int_{0}^{t} e^{A p(s)} d s-T_{0}\left(e^{A p(s)}-1\right) \\
E(t) & =E_{0}-\gamma\left(T_{0}+C_{0}\right) t+T_{0}(\beta+\gamma) \int_{0}^{t} e^{A p(s)} d s-\gamma u_{*} A T_{0} \int_{0}^{t} \int_{0}^{r} e^{A p(s)} d s d r(15) \\
(t & \in[0, \tau])
\end{aligned}
$$

where

$$
p(t)=\int_{0}^{t} u(s) d s
$$

For simplicity we normalize the technology stock $T(t)$ by its initial value $T_{0}$ and put

$$
x(t)=\frac{T(t)}{T_{0}}=e^{A p(t)} \quad(t \in[0, \tau]) ;
$$


introduce an auxiliary variable $y(\cdot)$ :

$$
y(t)=\int_{0}^{t} e^{A p(s)} d s \quad(t \in[0, \tau])
$$

In terms of the variables $x, y$ and based on Lemma 1 , we represent problem $\mathrm{A}$ as the following optimal control problem B:

Problem B: find a couple $(\tau, u(\cdot))$ that

$$
\begin{aligned}
W_{1}[\tau, u] & =\int_{0}^{\tau} e^{-\rho t} \ln x(t) d t+\frac{e^{-\rho \tau}}{\rho}\left[\ln x(\tau)+\frac{A \bar{u}+\lambda \ln a}{\rho}\right] \rightarrow \max _{\tau \geq 0, u(\cdot)}, \\
\dot{x}(t) & =A u(t) x(t), \quad x(0)=1 \\
u(t) & \in\left[0, u_{*}\right], \\
\dot{y}(t) & =x(t), \quad y(0)=0, \\
E_{0} & -\gamma_{0} \tau+\gamma_{2} y(\tau)-\gamma_{1} \int_{0}^{\tau} y(s) d s=E_{*}, \\
(t & \in[0, \tau]),
\end{aligned}
$$

where

$$
\begin{aligned}
\gamma_{0} & =\gamma\left(C_{0}+T_{0}\right) \\
\gamma_{1} & =\gamma u_{*} A T_{0}, \\
\gamma_{2} & =T_{0}(\beta+\gamma) .
\end{aligned}
$$

In what follows we assume that the probability of one hazard, $\lambda$ as well as the percentage loss $(1-a)$ from one hazard are large enough. Namely we introduce the following

High Risks Assumption: $A \bar{u}+\lambda \ln a<0$.

Figure 2 illustrates the area of admissible values of parameters $a$ and $\lambda$ under High Risks Assumption.

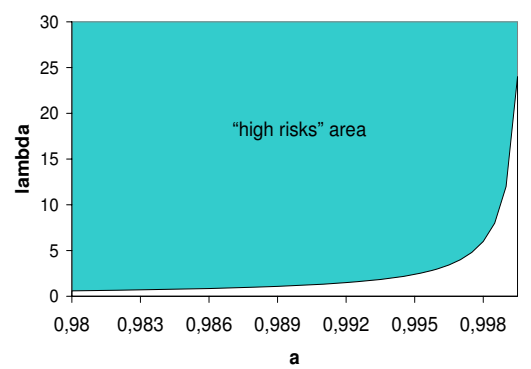

Figure 2: "High risks" area in terms of $a$ and $\lambda$.

Lemma 2 Let High Risks Assumption be satisfied. Let $(\tau, u(\cdot))$ be an arbitrary couple in which $u(t)(t \in[0, \tau])$ is a control in problem $B, \tau \geq 0$. Let $y(t)(t \in[0, \tau])$ be a 
corresponding solution of (20) and

$$
E_{0}-\gamma_{0} \tau+\gamma_{2} y(\tau)-\gamma_{1} \int_{0}^{\tau} y(s) d s<E_{*}
$$

Then there exist $a \hat{\tau} \geq \tau$ and a control $\hat{u}(t)$ extending $u(t)$ to $t \in[0, \hat{\tau}]$, that

1)

$$
E_{0}-\gamma_{0} \hat{\tau}+\gamma_{2} \hat{y}(\hat{\tau})-\gamma_{1} \int_{0}^{\hat{\tau}} \hat{y}(s) d s=E_{*}
$$

where $(\hat{x}(t), \hat{y}(t))$ is the solution of (20) corresponding to $\hat{u}(t)(t \in[0, \hat{\tau}])$; and

2)

$$
W_{1}[\hat{\tau}, \hat{u}] \geq W_{1}[\tau, u]
$$

Proof. Let $\hat{u}(t)=0$ for $t>\tau$. Let us show that $W_{1}[t, u]$ grows as $t$ grows, starting at $t=\tau$. Indeed, taking the derivative we get

$$
\frac{\partial W_{1}[t, u]}{\partial t}=\frac{e^{-\rho t}}{\rho}[A \bar{u}+\lambda \ln a]
$$

which is positive by High Risks Assumption. Taking into account the fact that $E(t)$ grows (see Remark 1) we come to the conclusion of the lemma.

The following theorem provides the main result of this section.

Theorem 2 If a couple $(\tilde{\tau}, \tilde{u}(\cdot))$ is a solution of Problem $B$ then the control $\tilde{u}(\cdot)$ has necessarily the single switching point of the max-min type, i.e.,

$$
\tilde{u}(t)=\left\{\begin{array}{cc}
u_{*}, & t \in[0, \xi) \\
0, & t \in[\xi, \tilde{\tau}]
\end{array}\right.
$$

for some $\xi \in[0, \tilde{\tau}]$.

Proof. 1. Suppose the contrary: let an optimal couple $(\bar{\tau}, \bar{u}(\cdot))$ be not of the max-min type. $\bar{y}(\cdot)$ the solution of $(20)$ corresponding to $\bar{u}(t)$. We assume that a trivial control $u(t)=1(t \in[0, \bar{\tau}])$ is not optimal in problem B, i.e., $\bar{y}>\bar{\tau}$. Let us fix $\bar{y}=\bar{y}(\bar{\tau})$.

2. Let us fix $\bar{\tau}$ and consider the following optimal control problem:

Problem C: find a control $u(\cdot)$ that

$$
\begin{aligned}
W_{2}[u] & =\int_{0}^{\bar{\tau}} y(s) d s \rightarrow \max _{u(\cdot)}, \\
\dot{x}(t) & =A u(t) x(t), \quad x(0)=1, \\
u(t) & \in\left[0, u_{*}\right], \\
\dot{y}(t) & =x(t), \quad y(0)=0, \quad y(\bar{\tau})=\bar{y}, \\
(t & \in[0, \bar{\tau}]) .
\end{aligned}
$$

Since $\bar{\tau}$ is fixed we set equivalent $W_{2}[u]=W_{2}[\bar{\tau}, u]$. By Lemma 3 (see Appendix C) the single optimal control in Problem C is

$$
\tilde{u}_{C}(t)=\left\{\begin{array}{cc}
u_{*}, & t \in[0, \xi), \\
0, & t \in[\xi, \bar{\tau}]
\end{array}\right.
$$

where $\xi$ is the single root of the equation

$$
e^{A u_{*} \xi}\left(\frac{1}{A u_{*}}+\bar{\tau}-\xi\right)-\frac{1}{A u_{*}}=\bar{y}
$$


3. Now let us consider the following optimal control problem:

Problem D: find a control $u(\cdot)$ that

$$
\begin{aligned}
W_{3}[u] & =\int_{0}^{\bar{\tau}} e^{-\rho t} \ln x(t) d t+\frac{e^{-\rho \bar{\tau}}}{\rho}\left[\ln x(\tau)+\frac{A \bar{u}+\lambda \ln a}{\rho}\right] \rightarrow \max _{u(\cdot)}, \\
\dot{x}(t) & =A x(t) u(t), \quad x(0)=1, \\
u(t) & \in\left[0, u_{*}\right], \\
\dot{y}(t) & =x(t), \quad y(0)=0, \quad y(\bar{\tau})=\bar{y}, \\
(t & \in[0, \bar{\tau}]) .
\end{aligned}
$$

Similar to the case of Problem C we set $W_{3}[u]=W_{3}[\bar{\tau}, u]$. By Lemma 4 (see Appendix C) $\tilde{u}_{C}(t)(25)$ is the single optimal control in Problem D.

4. Now let us consider the couple $\left(\bar{\tau}, \tilde{u}_{C}(\cdot)\right)$. Let $(\tilde{x}(t), \tilde{y}(t))$ be the solution of $(20)$ corresponding to $\tilde{u}_{C}(t)$.

The fact that control $\tilde{u}_{C}(\cdot)(25)$ is optimal in Problem $\mathrm{C}$ leads to

$$
E_{0}-\gamma_{0} \bar{\tau}+\gamma_{2} \tilde{y}(\bar{\tau})-\gamma_{1} \int_{0}^{\bar{\tau}} \tilde{y}(s) d s<E_{0}-\gamma_{0} \bar{\tau}+\gamma_{2} \bar{y}(\bar{\tau})-\gamma_{1} \int_{0}^{\bar{\tau}} \bar{y}(s) d s=E_{*} .
$$

The fact that the control $\tilde{u}_{C}(\cdot)(25)$ is optimal in Problem D and equality $\tilde{y}(\bar{\tau})=\bar{y}(\bar{\tau})$ lead to

$$
W_{3}\left[\tilde{u}_{C}\right]=W_{1}[\bar{\tau}, \tilde{u}]>W_{1}[\bar{\tau}, \bar{u}]
$$

(see the form of $W_{1}$ in (19)).

5. In a view of the inequalities (26) and (27), by Lemma 2 there exist a $\hat{\tau}>\bar{\tau}$ and a control $\hat{u}(t)$ extending $\bar{u}(t)$ to $[0, \hat{t}]$ such that $(22)$ and (23) hold. Now (23) and (27) show that the pair $(\bar{\tau}, \bar{u}(\cdot))$ is not optimal in Problem B. Thus we have arrived to a contradiction which proves the statement of the theorem.

From Theorems 1 and 2 follows

Theorem 3 If $(\tau, u(\cdot))$ is an optimal couple in the optimal economic growth problem (10) then

$$
u(t)= \begin{cases}u_{*}, & t \in[0, \xi), \\ 0, & t \in[\xi, \tau], \\ \bar{u}, & t \in(\tau, \infty),\end{cases}
$$

where $\xi \in[0, \tau]$.

\section{Global Calibration}

For practical simulation we provide a calibrated version of model (1) - (10) in global scale and run our model for 100 years time horizon starting from the year 2000. Table 4 provides calibrated values for the model's parameters. Some values are rather standard. For example, [Barro, Sala-i-Martin, 1995] and [Nordhaus, 1994] estimate that the production technology intensity and the discount factor as $\rho=0.03$ year $^{-1}$ and $A=4$ year $^{-1}$, respectively.

Here we restrict GHG to the main contributor in global warming - carbon dioxide $\mathrm{CO}_{2}$. In the year $2000 \mathrm{GDP}$ value $Y_{0}=26.7$ tril. US dollars and $\mathrm{CO}_{2}$ atmospheric concentration $E_{0}=262 \mathrm{ppm}$ can easily be found in economic-environmental databases - see, e.g., [IPCC, 2007]; the size of the production technology stock in the year 2000 , $T_{0}=6.6$ tril. US dollars, is calculated via (6). 


\begin{tabular}{|c|c|}
\hline 2000 year & Production technology intensity with respect to GDP \\
\hline$\theta=2100$ year & GDP elasticity with respect to production technology \\
\hline$A=4$ year $^{-1}$ & Production technology intensity \\
\hline$u_{*}=0.003$ & GDP fraction to be invested for technology development \\
\hline$\rho=0.03$ year $^{-1}$ & Discount factor \\
\hline$\beta=0.8 \mathrm{Gt} /($ year*tril. US dollars $)$ & Production technology intensity with respect to emissions \\
\hline$\lambda=3.5$ year $^{-1}$ & Expected (mean) value for annual number of catastrophes \\
\hline$a=0.9996$ & Not damaged fraction of GDP as a result of each catastrophe \\
\hline$Y_{0}=26.7$ tril. US dollars & Initial GDP \\
\hline$T_{0}=6.7$ tril. US dollars & Initial production technology \\
\hline$C_{0}=0$ & Initial cleaning technology \\
\hline$E_{0}=262 \mathrm{ppm}$ & Initial $\mathrm{CO}_{2}$ atmospheric concentration \\
\hline
\end{tabular}

Table 1: Calibrated values for the model's parameters.

The initial size of the cleaning technology stock can hardly be well estimated; we assume its value in the year 2000 to be negligibly small and put $C_{0}=0$.

We estimate the maximum resource for investment, $u_{*}$ assuming that in the period preceding 2000 business as usual (BAU) strategy of investment in production has been implemented. In other words, investment in cleaning has been insufficient for substantial growth of its stock which has led to the exponential GDP growth $Y(t)=Y_{0} e^{A u_{*}(t-2000)}$ (1), (6) for $t \leq 2000$, where $Y_{0}$ refers to the production technology stock in the year 2000. Evolving the past century world GDP statistics, available, e.g., in [Maddison, 1995] we regress $Y$ on $t$ and get $u_{*}$ equal $0.3 \%$.

When calibrating the $\mathrm{CO}_{2}$ growth function $\beta T-\gamma C(4)$ we identify $\dot{E}(t)$ with emissions $e(t)$ ignoring natural adaptation effects. Under the assumption on BAU strategy of investment in production implemented in the period preceding 2000 , i.e., for $t<2000$ we put emission function as $e(t)=\beta T(t)=\beta T_{0} e^{A u_{*}(t-2000)}$. We take data on global $C O_{2}$ emissions from [Marland et al, 2007] (http://cdiac.ornl.gov/trends/emis/em_cont.htm) provide regression $e$ on $t$ and get value for $\beta$ as $0.8 \mathrm{Gt} /$ (year $*$ tril. US dollars).

In trying to assess the Poisson distribution (8) parameters, $\lambda$ and $a$, we come across with a serious difficulty which one rather often appearing when calibrating continuous effects as discrete ones. Namely, the nature of the impact of the increase in atmospheric GHG on natural hazards is not very well explored. However, both the frequency of and the damage from them are expected to increase gradually while GHG concentration increases. Instead, in this work we simplify this complex gradual dependence into a switch between two extreme modes: a "safe" mode when no hazards occur (if $E(t)<E_{*}$ ) and a "dangerous" mode when hazards occur with a constant frequency $\lambda$ and damage $1-a$ (if $E(t)>E_{*}$ ). We assume that initially (in the year 2000 ) the system is in the "safe" mode. The latter assumption implies that the frequency and loss parameter values, $\lambda$ and $a$, are to be calibrated for a period in which the impact of hazards is ignorably small. Nevertheless we find average loss from each catastrophe to be $0.02 \%$ and average number of catastrophes to be 9 per year for the "safe" mode [IPCC, 2001]. For simulations we will vary the values of $\lambda$ and $a$ around these estimates carrying out the sensitivity analysis of the model's outcome to the input uncertainty in Poisson process parameters.

On the same reason, it turns out to be not possible to calibrate the critical level $E_{*}$ and cleaning technology intensity $\gamma$ since the society has not come across with a "dangerous" mode of the environmental behavior so far. This fact adds $E_{*}$ and $\gamma$ to the number of uncertain parameters in the model. 


\section{Optimal GDP and optimal GHG}

Let us first calculate the optimal utility $W$ (12). Substituting the form of the optimal control $u(24)$ in the utility $W_{1}(19)$ we find that the optimal utility value has the form

$$
W=W[\xi, \tau]=\frac{e^{-\rho \tau}}{\rho^{2}}[A \bar{u}+\lambda \ln a]+\frac{A u_{*}}{\rho^{2}}-\frac{e^{-\rho \xi}}{\rho^{2}}+\frac{\ln Y_{0}}{\rho},
$$

here $\tau>0$ is the point in time at which the accumulated emission hits the critical level $E_{*}$ and $\xi \in[0, \tau]$ is the switching time for the optimal control in Problem B. The optimal couple $(\xi, \tau)$, determining the optimal control (24) maximizes $W[\xi, \tau]$ under the constraints $\xi \geq 0, \tau \geq \xi, E(\tau)=E_{*}$. Given a $\xi \geq 0$ we find $\tau=\tau(\xi)$ from

$$
\frac{\beta T_{0}}{A u_{*}}\left[e^{A u_{*} \xi}-1\right]-\gamma C_{0} \xi+\left(\beta T_{0} e^{A u_{*} \xi}-\gamma C_{0}\right)(\tau-\xi)-\gamma u_{*} Y_{0} e^{A u_{*} \xi} \frac{(\tau-\xi)^{2}}{2}=E_{*}-E_{0} .
$$

Hence, in the optimal couple $(\xi, \tau)$ we have $\tau=\tau(\xi)$ and $\xi$ is found as the solution to the one-dimensional optimization problem

$$
W[\xi, \tau(\xi)] \rightarrow \max _{\xi \geq 0}
$$

Now let us give and analyze the optimal paths in Problem A. From (24) we see that the pre-perturbed period $[0, \tau]$ is split into two sub-periods: a period of intense production $[0, \xi]$ and a subsequent period $(\xi, \tau]$, at which special abatement measures on reducing GHG emissions are implemented. In period $[0, \xi]$ the optimal production technology stock exponentially grows and after $t=\xi$ it stabilizes:

$$
T(t)= \begin{cases}T_{0} e^{A u_{*} t}, & t \in[0, \xi] \\ T_{0} e^{A u_{*} \xi}, & t \in(\xi, \tau] .\end{cases}
$$

The optimal GDP is developing proportionally to the production technology stock with a coefficient $A$.

In period $[0, \xi]$ cleaning technology develops according to BAU strategy. After $t=\xi$ it grows linearly:

$$
C(t)= \begin{cases}C_{0}, & t \in[0, \xi] \\ C_{0}+u_{*} Y_{0} e^{A u_{*} \xi}(t-\xi), & t \in(\xi, \tau] .\end{cases}
$$

In period $[0, \xi]$, because of exponentially increasing production and BAU cleaning, the atmospheric GHG stock grows exponentially with the rate $\beta T_{0} e^{A u_{*} t}-\gamma C_{0}$; in the subsequent period $(\xi, \tau]$ in spite of the fact that intense production is not being developed any more and all resources are invested in cleaning, the atmospheric GHG continue growing with the linearly decreasing rate $\beta T_{0} e^{A u_{*} \xi}-\gamma C_{0}-\gamma u_{*} Y_{0} e^{A u_{*} \xi}(t-\xi)$ until they reach the critical level $E_{*}$ at $t=\tau$ :

$$
E(t)= \begin{cases}E_{0}-\gamma C_{0} t+\frac{\beta T_{0}}{A u_{*}}\left[e^{A u_{*} t}-1\right], & t \in[0, \xi], \\ E(\xi)+\left[\beta T_{0} e^{A u_{*} \xi}-\gamma C_{0}\right](t-\xi)-\gamma u_{*} Y_{0} e^{A u_{*} \xi \frac{(t-\xi)^{2}}{2}}, & t \in(\xi, \tau] .\end{cases}
$$

As the society enters the "dangerous" zone, natural hazards start to occur randomly. The optimal trajectories for the production technology stock, GDP, cleaning technology stock and GHG stock become stochastic. Due to the structure of the Poisson distribution describing the occurance of natural hazards, we assess the values for these variables in nodes of a time grid only. We choose a time grid with a step $\delta$ (say, one year)

$$
\left\{t_{k}\right\}_{k=0,1, \ldots}: \quad t_{0}=\tau, \quad t_{k}=t_{0}+k \delta .
$$


In accordance with $(1),(6)$ for every realization $\left(w_{0}, \ldots, w_{k}\right)$ we get

$$
Y_{k+1}=w_{k} Y_{k}+A \bar{u} w_{k} Y_{k} \delta=w_{k} Y_{k}[1+A \bar{u} \delta],
$$

hence

$$
Y_{k}=Y_{\tau}(1+A \bar{u} \delta)^{k} \Pi_{i=0}^{k-1} w_{i}=Y_{\tau}(1+A \bar{u} \delta)^{k} a^{\eta_{0}+\ldots+\eta_{k}},
$$

where $Y_{\tau}=Y_{0} e^{A u_{*} \xi}$, is the value of the optimal GDP at time $\xi$ the point of leaving a "safe" zone, $\eta_{0}, \ldots, \eta_{k}$ are numbers of catastrophes which occur in each year up to the year $t_{k}$. The latter formula holds because due to (8) the damage ratio each year does not depend on the number of the year $i$ and is given by $1-w_{i}=1-a^{\eta_{i}}$. Let us notice that the randomly damaged GDP in year $t_{k}(35)$ can be written as

$$
Y_{k}=Y_{\tau} e^{A u_{*} t_{k}} a^{\eta_{0}+\ldots+\eta_{k}}+O(\delta) .
$$

where $O(\delta) \rightarrow 0$.

For $\eta_{0}+\ldots+\eta_{k}=j(j=0,1, \ldots)$ formula (35) defines a spectrum of the optimal GDP at year $t_{k}$

$$
Y_{k j}=Y_{\tau}(1+A \bar{u} \delta)^{k} a^{j} \quad(j=0,1, \ldots)
$$

with corresponding probabilities

$$
P_{k j}=P\left[Y_{k}=Y_{k j}\right]=\frac{(\lambda \delta k)^{j}}{j !} e^{-\lambda \delta k} \quad(j=0,1, \ldots) .
$$

From (36) we see that in the year $t_{k}$ natural hazards reduce the deterministic annual GDP $Y_{\tau} e^{A \bar{u} t_{k}}$ for the fraction $1-a^{\eta_{0}+\ldots+\eta_{k}}$.

The expected optimal GDP is then

$$
\begin{aligned}
\mathbf{E}\left[Y_{k}\right] & =Y_{\tau}(1+A \bar{u} \delta)^{k} \mathbf{E}\left[a^{\eta_{0}+\ldots+\eta_{k}}\right] \\
& =Y_{\tau}(1+A \bar{u} \delta)^{k} \sum_{i=0}^{\infty} a^{i} \frac{(\lambda \delta k)^{i}}{i !} e^{-\lambda \delta k} \\
& =Y_{\tau}(1+A \bar{u} \delta)^{k} e^{-\lambda \delta k(1-a)} .
\end{aligned}
$$

Let us notice that the production technology stock obeys to the same probabilistic distribution as the GDP $Y$, i.e.,

$$
\begin{aligned}
T_{k j} & =T_{\tau}(1+A \bar{u} \delta)^{k} a^{j} \quad \text { with the probability given by (38), } \\
\mathbf{E}\left[T_{k}\right] & =T_{\tau}(1+A \bar{u} \delta)^{k} e^{-\lambda \delta k(1-a)} .
\end{aligned}
$$

Since $u(t)=u_{*}$ in the "unsafe" mode, from (2) we get that the cleaning technology stock remains constant, i.e., $C_{k}=C_{\tau}$.

The GHG stock in year $t_{k}$ and its expectation are given by

$$
\begin{aligned}
E_{k} & =E_{\tau}-\gamma k \delta C_{\tau}+\beta \delta T_{\tau} \sum_{i=0}^{k}(1+A \bar{u} \delta)^{i} a^{\eta_{0}+\ldots+\eta_{i}}, \\
\mathbf{E}\left[E_{k}\right] & =E_{\tau}-\gamma k \delta C_{\tau}+\beta \delta T_{\tau} \frac{(1+A \bar{u} \delta)^{k+1} e^{-\lambda \delta(1-a)(k+1)}-1}{(1+A \bar{u} \delta) e^{-\lambda \delta(1-a)}-1} .
\end{aligned}
$$

In section Sensitivity analysis one can find a calibrated version of model (1) - (10), numerically calculated optimal trajectories for the state variables and sensitivity analysis of the model's output to the uncertain parameters. 


\section{Sensitivity Analysis}

We us note that the life of the modelled system has two important time points, $\xi$ and $\tau$, switching time from intense production to intense cleaning, and starting time of catastrophes, respectively. Let us analyze them in terms of input uncertainties in $\beta, E_{*}, \lambda$, and $a$.

First, let us specify the procedure of maximizing $W(29)$. We find $\tau(\xi)$ from (30). Notice that the longer the period of intense production $[0, \xi]$, the shorter the period of intense cleaning $[\xi, \tau]$, and, moreover, the shorter the whole "safe" mode period $[0, \tau]$.

Let us notice also that the switching $\xi$ is constrainted from above and from below. On one hand, the duration of intense production period, $\xi$, can not be too long - it is limited by the condition $\xi \leq \tau$. On the other hand, $\xi$ should be long enough to guarantee that by the time $\tau$ GHG concentration $E(t)$ approaches the critical level $E_{*}$. Therefore,

$$
\xi \in\left[\xi_{\min }, \xi_{\max }\right]
$$

where

$$
\begin{aligned}
& \xi_{\text {min }}=\max \left\{0, \frac{1}{A u_{*}} \ln \frac{2 \gamma A u_{*}\left(E_{*}-E_{0}+\frac{\beta T_{0}}{A u_{*}}\right)}{\beta T_{0}(\beta+2 \gamma)}\right\}, \\
& \xi_{\text {max }}=\frac{1}{A u_{*}} \ln \left[1+\left(E_{*}-E_{0}\right) \frac{A u_{*}}{\beta T_{0}}\right] .
\end{aligned}
$$

Notice then, that the assumption (3) implies $\xi_{\min }=0$. Finally, we maximize $W(29)$ as $\xi \in\left[0, \xi_{\max }\right]$. From (43) we see that the area of admissible $\xi$ depends neither on $a$ and $\lambda$, nor on $\gamma$, logariphmically expanding with respect to $E_{*}$ (see Figure 43 ). Thus, if $E_{*}$ equals to $393 \mathrm{ppm}\left(1,5\right.$ times of $\left.E_{0}\right)$ one will choose the optimal switching time between the year 2000 and the year 2022, whereas if $E_{*}$ equals to $524 \mathrm{ppm}$ (doubled value of $E_{0}$ ) the upper limit for the interval for choosing the optimal switching time becomes 2039.

Now let us discuss the optimal choice of investment policy in the "safe" mode, i.e., the optimal time moments $\xi$ and $\tau$. The simulations show that there may be two principally different situations.

(i) Low damages from catastrophes and high cost of cleaning.

The aggregated damage of natural catastrophes over the whole perturbed period is less than the aggregated loss in the GDP due to special investment in cleaning. It means that it is not profitable to develop cleaning technology and the optimal investment strategy prescribes to allocate all resources in developing production. Then optimal time of starting catastrophes is given by $\tau=\xi=\xi_{\max }$. As we already mentioned, $\tau$ depends neither on $a$ and $\lambda$, nor on $\gamma$, logariphmically growing with respect to $E_{*}$ (see Figure 6).

Both the optimal GDP and the optimal GHG concentration grow exponentially if $t \in[0, \tau]$ (see (31) and (33) $)^{4}$ independently on $E_{*}, \gamma, \lambda$ and $a$. The expected GDP and the expected GHG concentration values for $t>\tau$ exponentially depend on $-\lambda(1-a)$ (see (39) and (41)). Figure 6 illustrates.

(ii) High damages from catastrophes and high cleaning efficiency.

The cleaning technology stock slows down the exponential growth of GHG concentration in the atmosphere and postpones the time when the system enters the "unsafe"

\footnotetext{
${ }^{4}$ Let us remind that in our simulations $C_{0}=0$.
} 
mode and catastrophes start to damage the fraction of the GDP. Abridgement of the perturbed period leads to decrease of the aggregated GDP losses and hence cleaning becomes profitable. Then the optimal investment strategy prescribes to start cleaning right in beginning of the modeling period. Then and $\xi=0$,

$$
\tau=\frac{\beta T_{0}-\sqrt{\beta^{2} T_{0}^{2}-2 \gamma u_{*} Y_{0}\left(E_{*}-E_{0}\right)}}{\gamma u_{*} Y_{0}} .
$$

From the latter formula we see that the optimal time for starting natural catastrophes $\tau$ depends sensitively on $E_{*}$ : the higher critical level of GHG concentration (i.e., the bigger the "safe" mode), the later catastrophes start to occur with increasing return to scale. The optimal time for starting natural catastrophes $\tau$ is rather insensitive to $\gamma$, but the increase the cost of cleaning technology leads to a slight increase in $\tau$. Comparison with the case (i) shows that catastrophes start later in case (ii) then in case (i) for all values of critical GHG level $E_{*}$ (see Figure 6).

Since all the investment is allocated in developing cleaning, the optimal GDP does not grow keeping its initial value $Y_{0}$ during the whole "safe" mode (31). The optimal cleaning technology stock grows linearly independently on $E_{*}, \gamma, \lambda$ and $a$ (32). Because of that, the optimal GHG concentration grows, and its rate is negatively affected by $\gamma$ (see (33)).

The expected GDP dramatically falls down damaged by catastrophes (39). Thanks to that the expected GHG concentration values stabilizes as soon as the system passes $t=\tau$ (41). Figure 6 illustrates.
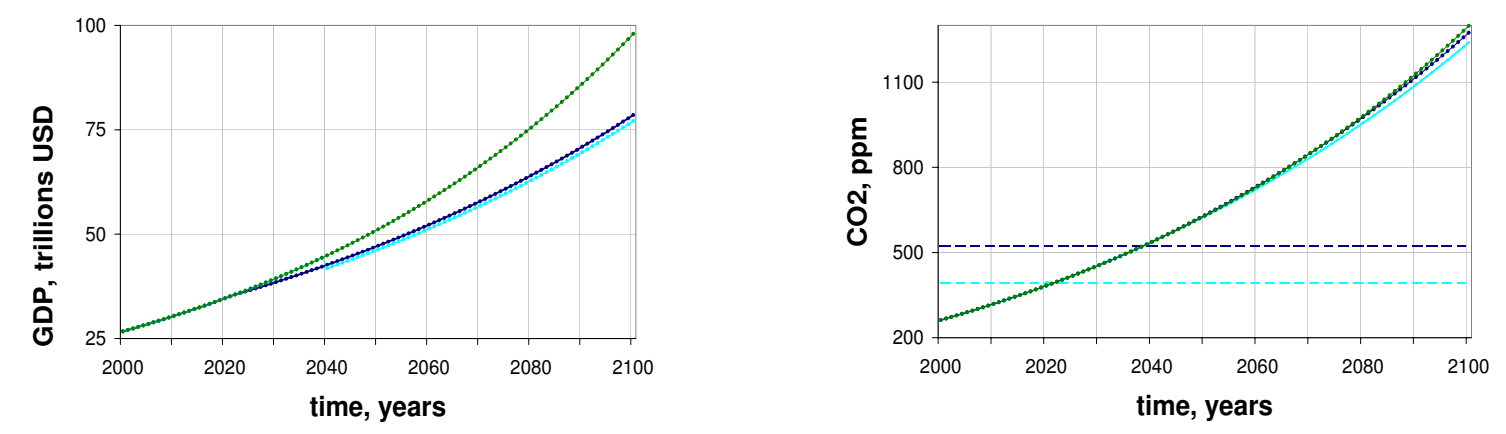

Figure 3: Case (i): optimal GDP trajectories (left plot) and optimal trajectories for GHG concentration in the atmosphere (right plot). The upper, dark-green curves correspond to $Y(t)$ and $E(t)$ trajectories in case of BAU production. The dark-blue and light-blue curves correspond to optimal $Y(t)$ and $E(t)$ for $E_{*}=1,5 E_{0}=393 \mathrm{ppm}$ and $E_{*}=2 E_{0}=524 \mathrm{ppm}$, respectively. Parameters' values: $\gamma=0,1 \mathrm{ppm} /$ year*tril.US dollars, $a=0,9996, \lambda=3,5$. 

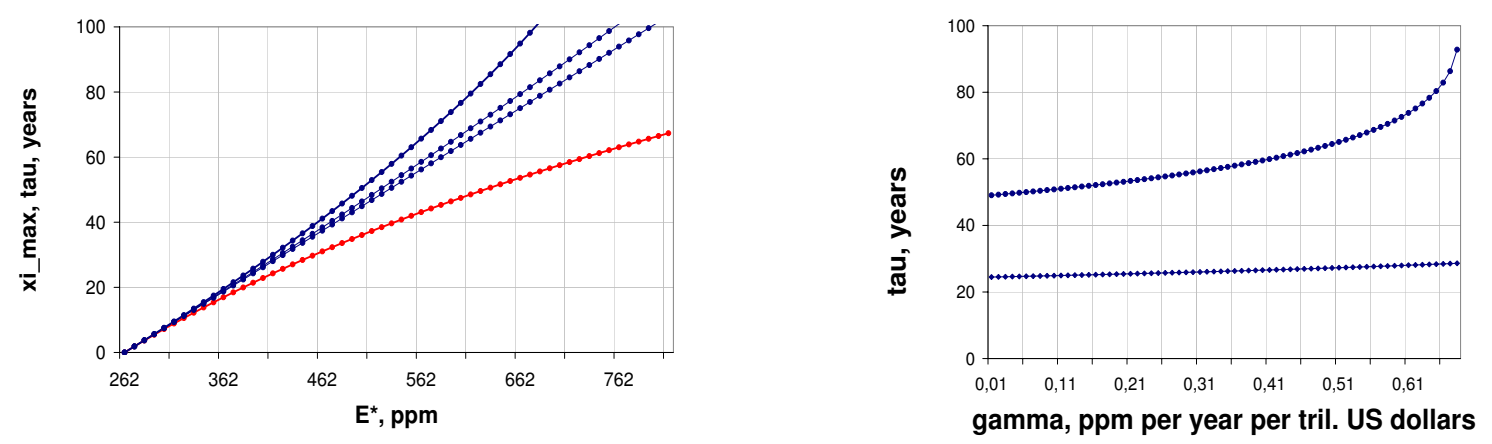

Figure 4: Optimal time moments of starting catastrophes. The left plot gives values for $\tau$ with respect to uncertain $E_{*}$ for the case (i) - the lower red curve, and for the case (ii) - three dark-blue curves, the upper of which corresponds to $\gamma=0.3 \mathrm{ppm} /$ year*tril.US dollars, the middle curve corresponds to $\gamma=0.1 \mathrm{ppm} /$ year*tril.US dollars, the lower curve corresponds to $\gamma=0.01 \mathrm{ppm}$ /year*tril.US dollars. The right plot gives values for $\tau$ in case (ii) with respect to uncertain $\gamma$ for $E_{*}=1,5 E_{0}=526 \mathrm{ppm}$ (the lower curve) and $E_{*}=2 E_{0}=526 \mathrm{ppm}$ (the upper curve).

\section{Discussion}

Let us start this section with discussion of what in fact new do we learn from explicit modeling of random hazards and their damages. Why not restrict ourselves to expected (mean) damages and cancel complicated stochastic dynamics and optimization in this problem? Generally speaking, these two ways are about an order of taking an expectation and non-linear instantaneous utility $f(Y)$. Namely, if case we consider stochastic dynamics, the utility to be maximized have a form $E\left[\int_{0}^{\infty} e^{-\rho t} f(Y(t)) d t\right]$ whereas in case of equivalent deterministic dynamics the utility takes a formula $\left.\int_{0}^{\infty} e^{-\rho t} f(E[Y(t)]) d t\right]$. Owing to non-linearity, not only values of these two functionals are be different for the same control which determines $Y(t)$, but also their properties related to optimization may not provide the same optimizator for both functionals ${ }^{5}$.

Second concern with respect to the results presented in this paper is a choice of Poisson distribution for the number of catastrophes in "unsafe" zone. Poisson distribution is often used for modeling events which occur with a known average rate, and which are independent on the time since the last event. A classic example is the nuclear decay of atoms. At the same time, it leads to a rather strong assumption on independence of the number catastrophes on the current level of atmospheric GHG provided the system exceeds the critical level $E_{*}$. On such a middle time horizon as 20-50 years we might accept that as a zero approximation. A way to overcome this problem might be in introducing a number of critical levels $E_{*}^{1}, E_{*}^{2}, \ldots, E_{*}^{n}$, and corresponding "unsafe" zones. In each zone the random number of natural hazards is distributed according to Poisson distribution which should have specific parameters' values $(\lambda$ and $a)$ : both the damage and the mean annual number of catastrophes should increase with an increase of $E_{*}^{i}$.

Let us summarize results which are obtained in this work:

\footnotetext{
${ }^{5}$ For precise quantitative estimate of the difference in consequences of optimal decisions made by means of these two different functionals, one should specially consider the corresponding deterministic optimization problem which is out of the goals of this paper.
} 

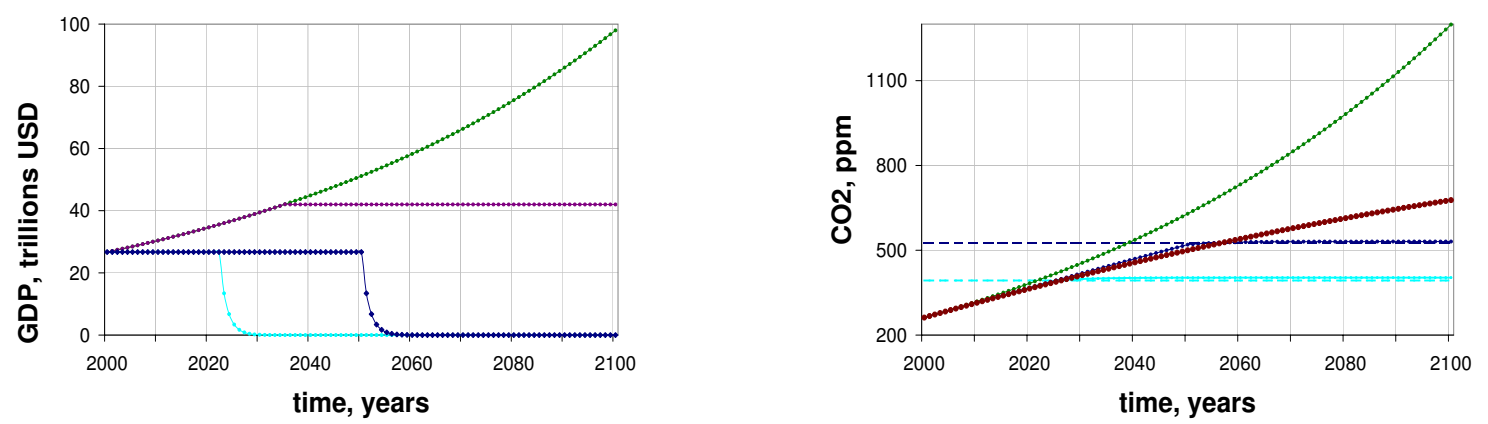

Figure 5: Case (ii): optimal GDP trajectories (left plot) and optimal trajectories for GHG concentration in the atmosphere (right plot). The upper, dark-green curves correspond to $Y(t)$ and $E(t)$ trajectories in case of BAU production. The dark-blue and light-blue curves correspond to optimal $Y(t)$ and $E(t)$ for $E_{*}=1,5 E_{0}=393 \mathrm{ppm}$ and $E_{*}=2 E_{0}=524 \mathrm{ppm}$, respectively. The dark-red curve on the right plot illustrates $E(t)$ trajectory in case of intense cleaning. Parameters' values: $\gamma=0,1 \mathrm{ppm} /$ year*tril.US dollars, $a=0,95, \lambda=14$.

1) We considered a one-sector economic growth model with production technology as a key driver of the economy and cleaning technology which is used for retraining greenhouse gases from the atmosphere. The important factor in the model - random natural hazards damaging the current GDP provided the atmospheric GHG level is high. We choose the utility as an expectation of the integrated discounted consumption. We formulated a problem of optimization of the economic growth on the infinite time horizon with respect to the utility. The optimal investment policy in production and cleaning is to be found.

2) We found the analytic structure of the optimal investment. It turns out that one should switch an optimal control mode twice. One should start from intense developing production providing zero investment in cleaning. GHG concentration is growing exponentially at that stage. The first switching point, $\xi$, opens a period of intense cleaning when the rate of increase of GHG in the atmosphere slows down. However at a time moment $\tau$ the system enters the "unsafe" mode, and catastrophes start to occur. In this period one invests all admissible resources in production.

3) We calibrated the model based on data available. Since the "unsafe" mode is only assumed to happen in future, we reveal uncertainty in values of the critical level $E_{*}$ of GHG concentrations in the atmosphere above which catastrophes affect the GDP significantly, of the cleaning efficiency, as well as of distribution parameters of random hazards.

4) It turns out that depending on the correlation between parameters of random catastrophes, $a$ and $\lambda$ and costs and efficiency of cleaning $\gamma$, two extreme case may hold. The first corresponds to the case of law damages from catastrophes and high cost of cleaning. Then the damage from catastrophes is less significant than investment in cleaning. Thus it is optimal to allocate all admissible resources for developing production, providing zero investment in cleaning. The system approaches the critical level $E_{*}$ fast, and, hence, catastrophes start to harm early which is nevertheless compensated by a relatively law damages.

The second case corresponds to completely opposite case - high damages from catas- 
trophes and high cleaning efficiency. In this case one should start cleaning as soon as possible, i.e., with the initial time moment of modeling. Because of high eventual damages from catastrophes it is optimal to postpone the time of starting catastrophes as long as possible. This idea implies zero investment in production and intensive developing of cleaning. Zero economic growth in the beginning is expected to be compensated by saving the GDP from catastrophes. Nevertheless, calculations show dramatic decrease of the GDP due to catastrophes in the "dangerous" mode.

The presented work is a step toward understanding how random natural hazards impact the technological development. Even under rather significant simplifications and strong assumptions made in this research, it reveals the eventual bifurcation of optimal dealing with economic growth harmed by natural hazards. Further quantitative and qualitative analysis of alternative hypothesis on both economic model and catastrophes regularities, as well as data analysis are needed to specify or refute them. 


\section{References}

[Barro, Sala-i-Martin, 1995] Barro, R.J., Sala-i-Martin, X. (1995). Economic growth, first ed. McGraw-Hill, New York, 1995.

[Hare, Meinshausen, 2006] Hare, B., Meinshausen, M. How Much Warming Are We Committed to and How Much Can Be Avoided?// Climatic Change, Vol. 75, 2006, pp. 111-149.

[IPCC, 2007] Fourth Assessment Report, The Intergovernmental Panel on Climate Change IPCC. Cambridge University Press, Cambridge UK, 2007.

[IPCC, 2001] Climate Change 2001: Synthesis Report, The Intergovernmental Panel on Climate Change IPCC. Cambridge University Press, Cambridge UK, 2007.

[Kainuma, 2006] Kainuma, M. Assessment of CO2 Reductions and Economic Impacts Considering Energy-saving Investments// Energy Journal. 2006.

[Keeling, Whorf, 2004] Keeling, C.D., Whorf, T.P. Atmospheric CO2 records from sites in the SIO air sampling network in Trends: A Compendium of Data on Global Change. Carbon Dioxide Information Analysis Center, Oak Ridge National Laboratory, U.S. Department of Energy, Oak Ridge, Tenn., U.S.A, 2004

[Keller et al, 2004] Keller, K., Bolker, B. M., Bradford, D. F. Uncertain Climate Thresholds and Optimal Economic Growth// Journal of Environmental Economics and Management, Vol. 48, Issue 1, July, 2004, pp. 723-741.

[Keywan et al, 2004] Keywan, R., Edward S.R., Margaret R.T., Schrattenholzer, L. and Hounshell, D., Technological learning for carbon capture and sequestration technologies, IIASA RR-04-012, 2004.

[Maddison, 1995] Maddison, A. Monitoring the World Economy. OECD Development Centre, Paris, 1995.

[Meinshausen et al, 2006] Meinshausen, M., Hare, B., Wigley, T., Van Vuuren, D., Elzen, M., Swart, R. Multi-Gas Emissions Pathways to Meet Climate Targets// Climatic Change, Vol. 75, 2006, pp. 151-194.

[Nordhaus, 1994] Nordhaus, W.D. Managing the Global Commons. The Economics of Climate Change. MIT Press, 1994.

[Nordhaus, Boyer, 2001] Nordhaus, W.D., and Boyer, J. Warming the World Economic Models of Global and Warming. MIT Press, 2001.

[Obersteiner et al, 2001] Obersteiner, M., et al. Managing Climate Risk// IIASA Interim Report, IR-01-051, December 2001.

[Marland et al, 2007] On-line trends: A compendium of data on Global Change. Global, Regional, and National Fossil Fuel CO2 Emissions: Marland, G., Boden, T. A. , and Andres, R. J.

http : //cdiac.ornl.gov/trends/emis/em_cont.htm

[O’Neill et al, 2005] O’Neill, B., Ermoliev, Yu., Ermolieva, T. Endogenous Risks and Learning in Climate Change Decision Analysis// IIASA Interim Report, IR-05-037, October 2005. 
[Rovenskaya, 2005] Rovenskaya, E. Sensitivity and cost-benefit analyses of emissionconstrained technological growth under uncertainty in natural emissions// IIASA Interim Report IR-05-051, October, 2005.

[Rovenskaya, 2006] Rovenskaya, E. A Model of Technological Growth under Emission Constraints// IIASA Interim Report, IR-06-021, May 2006.

[Toll, 1994] Toll, R. S. J. The Damage Costs of Climate Change: a Note on Tangibles and Intangibles, Applied to DICE// Energy Policy, Vol. 22, Issue 5, 1994. pp. 436-438. 


\section{A Proof of Theorem 1}

In this section we provide a proof for Theorem 1. First we discretesize model (1) - (7) on the perturbed period $t \geq \tau$. We introduce a discrete time grid

$$
\left\{t_{k}\right\}_{k=0,1, \ldots}: \quad t_{0}=\tau, \quad t_{k}=t_{0}+k \delta .
$$

with a small positive time step $\delta$.

According to (7) a random fraction of the production loss in each period $\left[t_{i}, t_{i+1}\right]$ becomes

$$
\omega_{i}=a^{\eta_{i}}
$$

where $\eta_{i}$ is a random number of natural hazards which occur over a time interval $\left[t_{i}, t_{i+1}\right]$. Let $\left(u_{0}, u_{1}, \ldots\right)$ be an approximation of a control $u^{1}(t)(t \in[\tau, \infty))(11)$. Model's dynamics (1) - (4) becomes

$$
\begin{aligned}
T_{i+1} & =w_{i} T_{i}+A u_{i} \omega_{i} T_{i} \delta, \quad T_{0}=T_{\tau}, \\
C_{i+1} & =C_{i}+A\left(u_{*}-u_{i}\right) \omega_{i} T_{i} \delta, \quad C_{0}=C_{\tau}, \\
E_{i+1} & =E_{i}+\left(\beta T_{i}-\gamma C_{i}\right) \delta, \quad E_{0}=E_{\tau}, \\
Y_{i} & =A w_{i} T_{i} \\
(i & =0,1, \ldots)
\end{aligned}
$$

where $T_{\tau}=T(\tau), C_{\tau}=C(\tau)$ and $E_{\tau}=E(\tau)$ are non-perturbed values of the production technology stock, cleaning technology stock and GHG stock at the moment $t=\tau$ at which the system leaves a non-perturbed zone.

Correspondingly taking into account (49), (46) the utility on the perturbed period $J^{1}$ (see (12) becomes

$$
\begin{aligned}
J^{1} & =\sum_{i=0}^{\infty} e^{-\rho\left(\tau+t_{i}\right)} \ln Y_{i} \delta \\
& =\sum_{i=0}^{\infty} e^{-\rho\left(\tau+t_{i}\right)} \ln \left(A w_{i} T_{i}\right) \delta \\
& =c_{*}^{1}(\delta)+e^{-\rho \tau} \sum_{i=0}^{\infty} e^{-\rho i \delta}\left[\ln \omega_{i}+\ln T_{i}\right] \delta
\end{aligned}
$$

where

$$
\begin{aligned}
c_{*}^{1}(\delta) & =\ln A e^{-\rho \tau} \sum_{i=0}^{\infty} e^{-\rho i \delta} \delta \\
& =\ln A e^{-\rho \tau} \frac{\delta}{1-e^{-\rho \delta}} .
\end{aligned}
$$

Note that

$$
c_{*}^{1}(\delta) \rightarrow \ln A \frac{e^{-\rho \tau}}{\rho} \quad \text { as } \quad \delta \rightarrow 0
$$

Clearly,

$$
\mathbf{E} J^{1}=c_{*}^{1}(\delta)+e^{-\rho \tau} \sum_{i=0}^{\infty} e^{-\rho i \delta} \mathbf{E}\left[\ln \omega_{i}+\ln T_{i}\right] \delta
$$


Let us specify the latter formula. From (46) we get

$$
\begin{aligned}
T_{i} & =T_{\tau} \prod_{k=0}^{i-1}\left(1+A u_{k} \delta\right) \prod_{k=0}^{i-1} \omega_{k} \\
& =T_{\tau} \prod_{k=0}^{i-1} e^{A u_{k} \delta} \prod_{k=0}^{i-1} \omega_{k}+O(\delta) \\
& =T_{\tau} e^{A p_{i}} \prod_{k=0}^{i-1} \omega_{k}+O(\delta)
\end{aligned}
$$

where

$$
p_{i}=\sum_{k=0}^{i-1} u_{k} \delta
$$

and

$$
O(\delta) \rightarrow 0 \quad \text { as } \quad \delta \rightarrow 0
$$

Hence,

$$
\ln T_{i}=\ln T_{\tau}+A p_{i}+\sum_{k=0}^{i-1} \ln \omega_{k}+O(\delta)
$$

and

$$
\mathbf{E} \ln T_{i}=\ln T_{\tau}+A p_{i}+\sum_{k=0}^{i-1} \mathbf{E} \ln \omega_{k}+O(\delta) .
$$

By (45) and (8)

$$
\begin{aligned}
\mathbf{E} \ln \omega_{k} & =\sum_{j=0}^{K} \frac{(\lambda \delta)^{j}}{j !} e^{-\lambda \delta} \ln a_{j} \\
& =\sum_{j=0}^{K} \frac{(\lambda \delta)^{j}}{j !} j e^{-\lambda \delta} \ln a \\
& =\sum_{j=1}^{K} \frac{(\lambda \delta)^{j}}{(j-1) !} e^{-\lambda \delta} \ln a \\
& =\lambda \delta \ln a+O(\delta) .
\end{aligned}
$$

Substituting in (53) we get

$$
\mathbf{E} \ln T_{i}=\ln T_{\tau}+A p_{i}+i \lambda \delta \ln a+O(\delta) .
$$

Coming back to (51) we find that

$$
\begin{aligned}
\mathbf{E} J^{1} & =c_{*}^{1}(\delta)+e^{-\rho \tau} \sum_{i=0}^{\infty} e^{-\rho i \delta} \mathbf{E}\left[\ln \omega_{i}+\ln T_{i}\right] \delta \\
& =c_{*}^{1}(\delta)+e^{-\rho \tau} \sum_{i=0}^{\infty} e^{-\rho i \delta}\left[\lambda \delta \ln a+\ln T_{\tau}+A p_{i}+(i-1) \delta \lambda \ln a+O(\delta)\right] \delta \\
& =c_{*}^{1}(\delta)+e^{-\rho \tau} \sum_{i=0}^{\infty} e^{-\rho i \delta}\left[\ln T_{\tau}+A p_{i}+i \delta \lambda \ln a\right] \delta+O(\delta) \\
& =C_{*}^{1}(\delta)+e^{-\rho \tau} \sum_{i=0}^{\infty} e^{-\rho i \delta}\left(A p_{i}+i \delta \lambda \ln a\right) \delta+O(\delta)
\end{aligned}
$$


where

$$
\begin{aligned}
C_{*}^{1}(\delta) & =c_{*}^{1}(\delta)+e^{-\rho \tau} \sum_{i=0}^{\infty} e^{-\rho i \delta} \ln T_{\tau} \delta \\
& =c_{*}^{1}(\delta)+\ln T_{\tau} e^{-\rho \tau} \frac{\delta}{1-e^{-\rho \delta}} .
\end{aligned}
$$

Note that

$$
C_{*}^{1}(\delta) \rightarrow \ln Y_{\tau} \frac{e^{-\rho \tau}}{\rho} \quad \text { as } \quad \delta \rightarrow 0 .
$$

From (55) we see that regardless the value of $\tau$ and the grid step $\delta, \mathbf{E} J^{1}$ approaches its maximum if each $p_{i}(i=0,1, \ldots)$ takes the maximum value. In other words $u_{k}=\bar{u}$ $(k=0,1, \ldots)$ brings the maximum value to $\mathbf{E} J^{1}$. Passage to a limit as $\delta \rightarrow 0$ finishes proving of the statement of the Theorem.

\section{B Proof of Lemma 1}

In this section we provide a proof for Lemma 1 . In other words we are aimed at specification of a form of the utility $W=J^{0}+\mathbf{E} J^{1}(12)$.

Let us specify $\mathbf{E} J^{1}$. From (55) (see Appendix A) by passage to a limit as $\delta \rightarrow 0$ we get

$$
\mathbf{E} J^{1}=\int_{\tau}^{\infty} e^{-\rho t} \mathbf{E} \ln Y(t) d t
$$

where

$$
\mathbf{E} Y(t)=Y_{\tau} e^{A p^{1}(t)+\lambda \ln a(t-\tau)} \quad(t \in[\tau, \infty)) .
$$

According to the Theorem 1 for $t \geq \tau u^{1}(t)=\bar{u}$ and

$$
p^{1}(t)=\bar{u}(t-\tau) \quad(t \in[\tau, \infty)) .
$$

Hence

$$
\begin{aligned}
\mathbf{E} J^{1} & =\int_{\tau}^{\infty} e^{-\rho t}\left[\ln Y_{\tau}+(A \bar{u}+\lambda \ln a)(t-\tau)\right] d t \\
& =\frac{e^{-\rho \tau}}{\rho}\left(\ln Y_{\tau}+\frac{A \bar{u}+\lambda \ln a}{\rho}\right) .
\end{aligned}
$$

Finally we obtain

$$
\begin{aligned}
W & =J^{0}+\mathbf{E} J^{1} \\
& =\int_{0}^{\tau} e^{-\rho t} \ln Y(t) d t+\frac{e^{-\rho \tau}}{\rho}\left(\ln Y_{\tau}+\frac{A \bar{u}+\lambda \ln a}{\rho}\right) .
\end{aligned}
$$

\section{Proofs of Lemma 3 and Lemma 4}

Lemma 3 The control optimal in the problem $C$ is

$$
\tilde{u}_{C}(t)=\left\{\begin{array}{cc}
u_{*}, & t \in[0, \xi], \\
0, & t \in(\xi, \bar{\tau}]
\end{array}\right.
$$

where $\xi$ is a root of an equation

$$
e^{A u_{*} \xi}\left(\frac{1}{A u_{*}}+\bar{\tau}-\xi\right)-\frac{1}{A u_{*}}=\bar{y} .
$$


Proof. We apply standard Pontryagin maximum principle to find a control optimal in problem C. Let $\psi_{1}(\cdot), \psi_{2}(\cdot)$ be adjoint variables. The Hamiltonian becomes

$$
H\left(x, y, \psi_{1}, \psi_{2}\right)=y+\psi_{1} A u x+\psi_{2} x
$$

and the Hamilton system supplying a solution of problem $\mathrm{C}$ is

$$
\begin{aligned}
\dot{\psi}_{1} & =-A u \psi_{1}-\psi_{2}, \quad \psi_{1}(\bar{\tau})=\bar{y} \\
\dot{\psi}_{2} & =-1, \\
\dot{x} & =A u x, \quad x(0)=1 \\
\dot{y} & =x, \quad y(0)=0, \quad y(\bar{\tau})=\bar{y} .
\end{aligned}
$$

The maximum condition becomes

$$
u(t)=\left\{\begin{array}{lll}
u_{*}, & \text { if } & \psi_{1}(t)>0 \\
\in\left[0, u_{*}\right], & \text { if } & \psi_{1}(t)=0 \\
0, & \text { if } & \psi_{1}(t)<0
\end{array}\right.
$$

From (62) we have

$$
\psi_{2}(t)=\psi_{2}^{0}-t
$$

with unknown initial value $\psi_{2}^{0}$. Then (61) becomes

$$
\dot{\psi}_{1}=-A u \psi_{1}+\left(t-\psi_{2}^{0}\right)
$$

and hence

$$
\psi_{1}(t)=\frac{1}{x(t)}\left[\psi_{1}^{0}+\int_{0}^{t} x(s)\left(s-\psi_{2}^{0}\right) d s\right] .
$$

Let us analyze the behavior of $\psi_{1}(t)$ in terms of its positiveness/negativeness. Since $x(t)>0$ for all admissible controls $u(\cdot)$ and all $t \in[0, \bar{\tau}]$ we focus on the expression in the square brackets only. Consider a function

$$
t \mapsto \phi_{1}(t)=\psi_{1}^{0}+\int_{0}^{t} x(s)\left(s-\psi_{2}^{0}\right) d s
$$

whose derivative is

$$
\dot{\phi}_{1}(t)=x(t)\left(t-\psi_{2}^{0}\right)
$$

and necesserely

$$
\phi_{1}(\bar{\tau})=0 .
$$

From (65) it follows that in problem $\mathrm{C}$ there is no special modes (controls for which $\psi_{1}(t)=0$ more then in one point). In other words an optimal control takes only its extreme values. Next, from (65) we see that the derivative $\dot{\phi}_{1}(t)$ changes its sign not more then in one point on $[0, \bar{\tau}]$. It means that necesseraly the following cases satisfy to the optimality conditions provided by Pontryagin maximum principle:

(i) $\psi_{1}^{0}>0$ and $\psi_{1}(t)>0$ for all $t<\bar{\tau}$ and $\psi_{1}(\bar{\tau})=0$; then

$$
u(t)=u_{*} \quad(t \in[0, \bar{\tau}]) ;
$$

(ii) $\psi_{1}^{0}>0$ and $\psi_{1}(t)$ changes its sign at some $t \in(0, \bar{\tau})$, namely, $\psi_{1}(t)>0$ for $t \in[0, \xi)$ and $\psi_{1}(t)<0$ for $t \in(\xi, \tau]$, and $\psi_{1}(\bar{\tau})=0$; then

$$
u(t)= \begin{cases}u_{*}, & t \in[0, \xi) \\ 0, & t \in[\xi, \bar{\tau}]\end{cases}
$$


(iii) $\psi_{1}^{0} \leq 0$ and $\psi_{1}(t)<0$ for all $t<\bar{\tau}$ and $\psi_{1}(\bar{\tau})=0$; then

$$
u(t)=0 \quad(t \in[0, \bar{\tau}]) .
$$

Control (iii), clearly, does not satisfy the condition $\bar{y}>\bar{\tau}$; depending on the value of $\bar{y}$ extreme control (i) may be not admissible in problem C. Otherwise it become a particular case of control (ii). Thus the two-stair control (ii) generalize the structure of an optimal control in problem $\mathrm{C}$. The switching time $\xi$ is determined in such a way that the edge condition $y(\bar{\tau})=\bar{y}$ holds which leads to the equation (60). The Lemma is proved.

Lemma 4 The control optimal in problem $D$ is $\tilde{u}_{C}(t)(t \in[0, \bar{\tau}])$ (59) (see Lemma 3).

Proof. First, let us notice that since the final time $\bar{\tau}$ is fixed, the goal function $W_{3}$ can be modified. Namely, we have

$$
\ln x(\bar{\tau})=\int_{0}^{\bar{\tau}} \frac{\dot{x}(s)}{x(s)} d s+\ln x_{0}=\int_{0}^{\bar{\tau}} A u(s) d s+\ln x_{0}
$$

and hence problem $\mathrm{D}$ is equivalent to the optimal control problem of maximization of the goal function

$$
W_{3}^{\prime}[u]=\int_{0}^{\bar{\tau}}\left[e^{-\rho t} \ln x(t)+A u(t)\right] d t
$$

under the same dynamics.

Similar to to the proof of the Lemma 3 we apply standard Pontryagin maximum principle to find a control optimal in the problem D. Let $\psi_{1}(\cdot), \psi_{2}(\cdot)$ be adjoint variables. The Hamiltonian becomes

$$
H\left(t, x, y, \psi_{1}, \psi_{2}\right)=e^{-\rho t} \ln x+A u+\psi_{1} A u x+\psi_{2} x
$$

and the Hamiltonian system supplying a solution of the problem $\mathrm{C}$ is

$$
\begin{aligned}
\dot{\psi}_{1} & =-A u \psi_{1}-\psi_{2}+\frac{R}{x^{2}}-\frac{e^{-\rho t}}{x}, \quad \psi_{1}(\bar{\tau})=\bar{y} \\
\dot{\psi}_{2} & =0, \\
\dot{x} & =A u x, \quad x(0)=1 \\
\dot{y} & =x, \quad y(0)=0, \quad y(\bar{\tau})=\bar{y} .
\end{aligned}
$$

The maximum condition becomes

$$
u(t)=\left\{\begin{array}{lll}
u_{*}, & \text { if } \quad \psi_{1}(t)>0 \\
\in\left[0, u_{*}\right], & \text { if } \quad \psi_{1}(t)=0 \\
0, & \text { if } \quad \psi_{1}(t)<0
\end{array}\right.
$$

From (67) we have

$$
\psi_{2}(t)=\psi_{2}^{0}
$$

with unknown initial value $\psi_{2}^{0}$. Then (66) becomes

$$
\dot{\psi}_{1}=-A u \psi_{1}+\left(\psi_{2}^{0}+\frac{e^{-\rho t}}{x}\right)
$$

and hence

$$
\begin{aligned}
\psi_{1}(t) & =\frac{1}{x(t)}\left(\psi_{1}^{0}+\int_{0}^{t}\left(e^{-\rho s}+\psi_{2}^{0} x(s)\right) d s\right) \\
& =\frac{1}{x(t)}\left(\psi_{1}^{0}-\frac{1-e^{-\rho t}}{\rho}+\psi_{2}^{0} y(t)\right) .
\end{aligned}
$$


Let us analyze the behavior of $\psi_{2}(t)$ in terms of its positiveness/negativeness. Since $x(t)>0$ for all admissible controls $u(\cdot)$ and all $t \in[0, \bar{\tau}]$ we focus on the expression in the square brackets only. Consider a function

$$
t \mapsto \phi_{2}(t)=\psi_{1}^{0}-\frac{1-e^{-\rho t}}{\rho}+\psi_{2}^{0} y(t)
$$

whose derivative is

$$
\dot{\phi}_{2}(t)=-e^{-\rho t}+\psi_{2}^{0} x(t) .
$$

and necessaraly

$$
\phi_{2}(\bar{\tau})=0 .
$$

Since $e^{-\rho t}$ decreases and $x(t)$ increases, from (70) we see that there may not be special modes in problem D. Next, from (70) we see that the derivative $\dot{\phi}_{2}(t)$ changes its sign not more then in one point on $[0, \bar{\tau}]$. It means that necesseraly the following cases satisfy to the optimality conditions provided by Pontryagin maximum principle:

(i) $\psi_{2}^{0} \leq 0$ and $\psi_{1}^{0}>0$ for all $t<\bar{\tau}$ and $\psi_{1}(\bar{\tau})=\bar{y}$; then

$$
u(t)=u_{*} \quad(t \in[0, \bar{\tau}]) ;
$$

(ii) $0<\psi_{2}^{0}<1$ and either $\psi_{1}(t)>0$ for all $t<\bar{\tau}$ and $\psi_{1}(\bar{\tau})=\bar{y}$, or $\psi_{1}(t)$ changes its sign at some $t \in(0, \bar{\tau})$, namely, $\psi_{1}(t)>0$ for $t \in[0, \xi)$ and $\psi_{1}(t)<0$ for $t \in(\xi, \bar{\tau}]$, and $\psi_{1}(\bar{\tau})=0$; then either

$$
u(t)=u_{*} \quad(t \in[0, \bar{\tau}])
$$

or

$$
u(t)= \begin{cases}u_{*}, & t \in[0, \xi) \\ 0, & t \in[\xi, \bar{\tau}]\end{cases}
$$

(iii) $\psi_{1}^{0}>1$ and $\psi_{1}(t)<0$ for all $t<\bar{\tau}$, and hence

$$
u(t)=0 \quad(t \in[0, \bar{\tau}]) .
$$

Similar to the case of Lemma 3 control (iii) does not satisfy to the condition $\bar{y}>\bar{\tau}$; depending on the value of $\bar{y}$ extreme control (i) may be not admissible in problem $\mathrm{C}$. Otherwise it become a particular case of control (ii). Thus the two-stair control (ii) generalize the structure of an optimal control in problem D. The switching time $\xi$ is determined in such a way that the edge condition $y(\bar{\tau})=\bar{y}$ holds which leads to the equation (60). The Lemma is proved. 